\title{
Longitudinal Mixed-Effect Model Analysis of the Association between Global and Tissue-Specific Brain Atrophy and Lesion Accumulation in Patients with Clinically Isolated Syndrome
}

\author{
M. Varosanec, T. Uher, D. Horakova, J. Hagemeier, N. N. Bergsland, M. Tyblova, Z. Seidl, M. Vaneckova, J. Krasensky, M.G. Dwyer,
}

E. Havrdova, and R. Zivadinov

\begin{abstract}
BACKGROUND AND PURPOSE: The relationship between lesion formation and brain atrophy development in the early phase of multiple sclerosis is unclear. We investigated the association between new lesion accumulation and brain atrophy progression in patients with clinically isolated syndrome over 48 months.
\end{abstract}

MATERIALS AND METHODS: Patients with clinically isolated syndrome $(n=210)$ were evaluated with 1.5 T MR imaging at baseline and at $6,12,24,36$, and 48 months as part of a multicenter observational study of early administration of intramuscular interferon $\beta$-la. Mixedeffect model analyses, adjusted for age, sex, and treatment status, investigated the association between accumulation of contrastenhancing and $\mathrm{T} 2$ lesions and brain-volume percent changes in a 48-month period.

RESULTS: In patients with clinically isolated syndrome, the average whole-brain volume decreased $2.5 \%$, the mean lateral ventricle volume increased 16.9\%, and a mean of 7.7 new/enlarging T2 lesions accumulated over the follow-up period. Patients with clinically isolated syndrome who showed greater percentages of change in whole-brain, white and gray matter, cortical, and lateral ventricle volumes over the follow-up period had more severe lesion outcomes at baseline (all $P<$.007). There were significant associations between decreased individual brain-volume measures at baseline and greater percentages of change during follow-up $(P<.05)$. We found a significant association between the total cumulative number of new/enlarging T2 lesions and the evolution of whole-brain $(P<.001)$, lateral ventricle $(P=.007)$, gray matter and thalamic $(P=.013)$, subcortical deep gray matter $(P=.015)$, and cortical $(P=.036)$ volumes over the follow-up period.

CONCLUSIONS: Lesion accumulation and brain-volume changes occur simultaneously in the early phase of clinically isolated syndrome. More severe lesion and brain-volume outcomes at baseline were associated with greater development of brain atrophy over the follow-up period in patients with clinically isolated syndrome.

ABBREVIATIONS: CIS = clinically isolated syndrome; $\mathrm{CE}=$ contrast-enhancing; $\mathrm{EDSS}=$ Expanded Disability Status Scale; $\mathrm{LV}=$ lesion volume; $\mathrm{SDGM}=$ subcortical deep gray matter

M ultiple sclerosis is a chronic inflammatory demyelinating disease that affects the central nervous system. Approximately $85 \%$ of patients with MS begin with the relapsing-remit-

Received October 31, 2014; accepted after revision December 26.

From the Buffalo Neuroimaging Analysis Center (M.V., T.U., J.H., N.B., M.G.D., R.Z.), Department of Neurology, University at Buffalo SUNY, Buffalo, New York; Department of Neurology and Center of Clinical Neuroscience (T.U., D.H., M.T., E.H.) and Department of Radiology (Z.S., M.V., J.K.), Charles University in Prague, First Faculty of Medicine and General University Hospital, Prague, Czech Republic; and IRCCS "Santa Maria Nascente" (N.B.), Don Gnocchi Foundation, Milan, Italy.

The Observational Study of Early Interferon $\beta$-la Treatment in High Risk Subjects after CIS (SET) study was supported by Czech Ministries of Education and Health (NT13237-4/2012, PRVOUK-P26/LFl/4, RVO-VFN64165/2012) and Biogen Idec.

Please address correspondence to Robert Zivadinov, MD, PhD, FAAN, Buffalo Neuroimaging Analysis Center, MRI Clinical Translational Research Center, Department of Neurology, School of Medicine and Biomedical Sciences, University at Buffalo, 100 High St, Buffalo, NY 14203; e-mail: rzivadinov@bnac.net

- Indicates open access to non-subscribers at www.ajnr.org

Indicates article with supplemental on-line photo. ting form. ${ }^{1}$ This event consists of an episode of neurologic disturbance known as a clinically isolated syndrome (CIS). The risk of progression to clinically definite MS is highest within the first 5 years of the initial event.

The development of brain atrophy is a well-known feature of MS. ${ }^{2}$ The general loss of brain tissue in MS derives from focal and diffuse damage, including loss of myelin and axons and Wallerian neurodegeneration. ${ }^{3}$ In recent years, there has been an increased focus on GM pathology in MS. This surge has been triggered by new histochemical and MR imaging techniques that enable improved detection of GM pathology. ${ }^{4}$ Recent studies have shown that global and regional GM atrophy at the first clinical event in patients with CIS is associated with conversion to clinically definite $\mathrm{MS}^{5-8}$

GM pathology in MS has become a focal point of research

http://dx.doi.org/10.3174/ajnr.A4330 
since the discovery that GM atrophy can predict clinical outcomes better than WM pathology.9-11 Weak-to-modest associations between the development of regional brain atrophy and changes in lesion number and lesion volume (LV) suggest that newly detected lesions, as well as progressive tissue damage in pre-existing lesions, contribute only partially to the loss of overall brain tissue. ${ }^{12,13}$ At this time, the importance of lesion formation in relation to brain atrophy development is unclear, especially at the earliest clinical stages of MS.

Against this background, the aim of the present study was to investigate the relationship between new/enlarging lesion formation and brain atrophy progression over 48 months in patients with CIS who presented with their first clinical event.

\section{MATERIALS AND METHODS Study Population}

This was an investigator-initiated, multicenter, prospective, observational clinical study in patients with CIS in which we investigated the evolution of clinical and MR imaging outcomes over 24 and 48 months. ${ }^{5,14-17}$

Inclusion criteria were age between 18 and 55 years and enrollment into the study within 4 months of the first clinical event. Criteria included diagnostic MR imaging showing $\geq 2$ T2-hyperintense lesions, an Expanded Disability Status Scale (EDSS) score of $\leq 3.5$, and $\geq 2$ oligoclonal bands in the CSF at the screening visit before the start of treatment. Exclusion criteria were a second relapse before the baseline examination, missing or invalid clinical or MR imaging follow-up information after the baseline examination, and pregnancy. Clinical visits were performed every 3 months, and disability, as measured by the EDSS, was assessed every 6 months. MR imaging was performed at baseline and at 6 , $12,24,36$, and 48 months.

All patients received the same treatment at baseline, which included $30 \mu \mathrm{g}$ of intramuscular interferon $\beta$-1a once per week. Necessary adjustments were made in the treatment for patients who presented with no or limited treatment effects, including the development of 2 relapses, a 6-month sustained EDSS progression of 1 point, or other clinical reasons. Each study subject was treated with 3-5 g of methylprednisolone after the first symptom before study entry, and a baseline MR imaging examination was performed at least 30 days after administration of the steroid. During the study, relapses were treated with 3-5 g of methylprednisolone.

The local ethics committees approved the study protocol, and each study subject gave written informed consent.

\section{MR Imaging Acquisition and Analysis}

MR imaging was performed at baseline and at 6, 12, 24, 36, and 48 months with a standardized protocol using the same $1.5 \mathrm{~T} \mathrm{MR}$ imaging scanner (Gyroscan; Philips Healthcare, Best, the Netherlands). Axial brain images were obtained by using FLAIR with a section thickness of $1.5 \mathrm{~mm}$ (TR, 11,000 ms; TE, $140 \mathrm{~ms}$; TI, 2600 ms; matrix size, $256 \times 181$; flip angle, $\left.90^{\circ}\right)$. Axial T1-weighted 3D T1 images were acquired with a section thickness of $1 \mathrm{~mm}$ (TR, 25 ms; TE, $5 \mathrm{~ms}$; matrix size, $256 \times 204$; flip angle, $30^{\circ}$ ). The FLAIR and 3D T1 images were contiguous. In addition, each patient underwent a postcontrast $\mathrm{T} 1$ spin-echo scan with a section thick- ness of $3 \mathrm{~mm}, 5$ minutes after contrast injection of a single dose of $0.1 \mathrm{mmol} / \mathrm{kg}$ of Gd-DTPA (TE, $12 \mathrm{~ms}$; TR, $450 \mathrm{~ms}$ ).

The image analysis included the cumulative number of new and enlarging T2 lesions, defined as the overall number of new and enlarging $\mathrm{T} 2$ lesions, and the cumulative number of contrast-enhancing (CE) lesions between all time points from baseline to 48 months. All lesion measures were performed by a single analyst (M.G.D.) who was blinded to the disease status of the patients. T2 and CE LVs were calculated by applying a semiautomated contouring-thresholding technique in Jim software (http://www.xinapse.com). ${ }^{18}$

Baseline whole-brain, GM, WM, cortical, and lateral ventricle volumes were calculated by using FSL Structural Imaging Evaluation of Normalized Atrophy Cross-sectional (SIENA/X; http:// fsl.fmrib.ox.ac.uk/fsl/fslwiki/SIENA). ${ }^{19}$ Subcortical deep gray matter (SDGM) — defined as the thalamus, globus pallidus, putamen, caudate, nucleus accumbens, hippocampus, and amygdala-volume was calculated by using the FMRIB Integrated Registration and Segmentation Tool (FIRST; http://fsl.fmrib.ox.ac.uk/fsl/ fslwiki/FIRST). ${ }^{20}$ All brain-volume measures were performed by a single analyst (N.B.) who was blinded to the disease status of the patients. Each baseline tissue volume was normalized for head size by using a skull-constrained registration to the standard MNI 152 template. Longitudinal percent changes in whole-brain, GM, WM, cortical, and lateral ventricle volumes were obtained by using direct methods of atrophy measurement. Briefly, whole-brain volume changes were measured by using the SIENA technique, ${ }^{21}$ whereas GM, WM, and lateral ventricle changes were calculated by using a longitudinal regularization of a hidden Markov random field model. ${ }^{22}$ Absolute and percent volume changes for the SDGM and thalamus at each time point were calculated.

\section{Statistical Analysis}

SPSS (version 21; IBM, Armonk, NY) and Statistica (version 10; StatSoft, Tulsa, Oklahoma) were used for all analyses. Because of non-normal distribution of the data, as assessed by using the Kolmogorov-Smirnov method, T2 and CE LVs and lesion numbers were logarithmically transformed.

Longitudinal linear mixed-effect models were used with random intercept for patients and adjusted for age, sex, and treatment status to describe temporal associations between baseline and follow-up MR imaging measures. Separate longitudinal linear mixed-effect models with a random intercept for patients, with interaction with time, were used to describe temporal associations between changes in individual MR imaging measures over the 48 months. The analyses included percent change values for whole-brain, GM, WM, lateral ventricle, cortical, SDGM, and thalamic volumes for each time point.

Mixed-effect model analyses investigated the association between MR imaging lesion outcomes, including the cumulative T2 and CE lesion activity, absolute T2 and CE LV changes, CE positivity (general occurrence of CE lesions during the study period), and the evolution of MR imaging brain volumetric percentage measures of global and tissue-specific volumes over 48 months.

The first mixed-effect model explored the association between the number of T2 and CE lesions, T2 and CE LVs, and CE positivity at baseline as the independent predictor variables with re- 
spect to the dependent outcome variables, which were global and tissue-specific brain-volume percent changes over the 48 months. To minimize the potential confounding effect of baseline brain volumes, we performed an additional confirmatory analysis adjusted also for baseline brain volumes.

After this analysis, we investigated the relationship between baseline MR imaging brain volumetric measures (independent variables) and the evolution of global and tissue-specific brainvolume percent changes over the 48 months (dependent variables).

Finally, we analyzed the association between lesion activity and brain-volume changes over the whole study period by comparing the total cumulative $\mathrm{T} 2$ and CE lesion activity and absolute T2 and CE LV changes over the 48 months with global and tissuespecific brain-volume percent changes over the same time period. To minimize the potential effect of baseline brain volume and LV, we performed an additional confirmatory analysis adjusted also for baseline brain volume and LV.

The results of the mixed-effect model analyses are presented for the whole study population. The Benjamini-Hochberg correction was used to minimize the false-discovery rate, and $P$ values of $<.05$ are considered significant. ${ }^{23}$

Table 1: Demographic, clinical, and MRI lesion and brain volumetric measures in patients with CIS over a 48-month period $(n=210)^{a}$

\begin{tabular}{lc}
\hline \multicolumn{1}{c}{ Measure } & Patient Data \\
\hline No. (\%) female & $139(66)$ \\
Age at onset, $y$ & $28.7 \pm 7.9$ \\
Time to baseline, days & $81.9 \pm 23.7$ \\
EDSS at baseline b $^{\mathrm{b}}$ & $1.7 \pm 0.7 ; 1.5(0.0-3.5)$ \\
EDSS at 48 mo & $1.8 \pm 0.9 ; 1.5(0.0-6.5)$ \\
Cumulative no. of & \\
$\quad$ Total new T2 lesions & $7.7 \pm 14.4 ; 3.0$ \\
$\quad$ New T2 lesions & $5.8 \pm 10.7 ; 2.0$ \\
$\quad$ Newly enlarging T2 lesions & $1.9 \pm 4.6 ; 0.0$ \\
Cumulative no. of new CE lesions & $1.5 \pm 6.1 ; 0.0$ \\
T2 lesion volume absolute change, $\mathrm{mL}$ & $-0.08 \pm 3.6 ;-0.1$ \\
CE lesion volume absolute change, $\mathrm{mL}$ & $-0.07 \pm 0.3 ; 0.0$ \\
WB volume, \% change & $-2.5 \pm 2.1 ;-2.0$ \\
GM volume, \% change & $-2.3 \pm 2.3 ;-2.0$ \\
WM volume, \% change & $-1.7 \pm 2.1 ;-1.4$ \\
Cortical volume, \% change & $-2.8 \pm 2.2 ;-2.6$ \\
Lateral ventricle volume, \% change & $16.9 \pm 14.2 ; 14.3$ \\
Total normalized SDGM volume, & $-3.6 \pm 3.6 ;-3.1$ \\
$\quad$ \% change & \\
Thalamic volume, \% change & $-4.6 \pm 4.2 ;-4.0$ \\
\hline
\end{tabular}

Note:-WB indicates whole brain.

a Unless otherwise indicated, all data are reported as mean \pm standard deviation; median.

${ }^{\mathrm{b}}$ Data in parentheses are ranges.

\section{RESULTS}

Demographic, Clinical, and MR Imaging Characteristics

Overall, 210 patients with CIS underwent clinical and MR imaging assessments and were included in the analysis over the 48 months of this study. Demographic, clinical, and MR imaging global and tissue-specific volumes and lesion characteristics over the 48 months are shown in Table 1. The mean age at onset was 28.7 years, and the median baseline EDSS score was 1.5. An average total of 7.7 new T2 lesions were accumulated over 48 months. Global and tissue-specific brain-volume percent changes showed average decreases of $2.5 \%$ in whole-brain, $2.8 \%$ in cortical, and $4.6 \%$ in thalamic volumes and an average increase of $16.9 \%$ in lateral ventricle volumes.

\section{Mixed-Effect Model Analysis Using Baseline Lesion Measures as Independent Outcome Variables and Brain- Volume Evolution Measures as Dependent Outcome Variables}

Table 2 shows the relationship between baseline lesion measures and the evolution of brain-volume percent changes over the 48 months. Patients with CIS who showed greater percent changes in whole-brain, WM, GM, cortical, and lateral ventricle volumes over the 48 months had a greater lesion activity at baseline (all $P<$ .007). The percent change in thalamic volume over the 48 months was associated with greater baseline T2 LV and lesion number $(P \leq .001)$ and baseline $\mathrm{CE}$ lesion number $(P=.018)$, whereas the percent change in SDGM volume was associated only with greater baseline CE lesion number $(P=.029)$.

Patients with CIS with the highest number of CE lesions at baseline progressed the most in whole-brain, cortical, and lateral ventricle volume changes over the 48 months (all $P<$.0001) (Fig 1). The association was somewhat less pronounced for thalamic volume $(P=.018)$.

Similarly, patients with CIS with the highest number of T2 lesions at baseline progressed the most in cortical, thalamic, and lateral ventricle volume changes (all $P<.0001$ ), and there was also an association with the whole-brain volume progression $(P=.01)$ (Fig 2).

The effects of baseline T2 and CE lesion numbers and T2 LV on whole-brain, WM, GM, cortical, and lateral ventricle volume changes persisted (all $P<.008$ ) even when adjusting the analyses for baseline brain volumes. In this confirmatory analysis, the percent change in thalamic volume over the 48 months was associated with greater baseline T2 LV and lesion number $(P<.001)$ and baseline $\mathrm{CE}$ lesion number $(P=.004)$, whereas the percent

Table 2: Relationship between MRI lesions at baseline and evolution of MRI brain volumetric measures in patients with CIS over a 48-month period ${ }^{\mathrm{a}}$

\begin{tabular}{|c|c|c|c|c|c|c|c|}
\hline \multirow[b]{2}{*}{ MRI Lesions at Baseline } & \multicolumn{7}{|c|}{ MRI Brain Volume \% Changes Over 48 mo } \\
\hline & WB & WM & GM & Cortical & Lateral Ventricle & SDGM & Thalamus \\
\hline T2 LV & $0.001^{\mathrm{b}}$ & $.001^{\mathrm{b}}$ & $<.001^{\mathrm{b}}$ & $.004^{\mathrm{b}}$ & $<.001^{\mathrm{b}}$ & .133 & $<.001^{\mathrm{b}}$ \\
\hline T2 LN & $.010^{b}$ & $.001^{b}$ & $<.001^{\mathrm{b}}$ & $<.001^{\mathrm{b}}$ & $<.001^{b}$ & .243 & $<.001^{b}$ \\
\hline CE positivity & $<.001^{\mathrm{b}}$ & $.007^{\mathrm{b}}$ & $.004^{b}$ & $.002^{b}$ & $<.001^{\mathrm{b}}$ & .292 & .517 \\
\hline CE LN & $<.001^{\mathrm{b}}$ & $<.001^{\mathrm{b}}$ & $<.001^{\mathrm{b}}$ & $<.001^{\mathrm{b}}$ & $<.001^{\mathrm{b}}$ & $.029^{b}$ & $.018^{b}$ \\
\hline CE LV & $<.001^{b}$ & $.001^{b}$ & $<.001^{b}$ & $<.001^{b}$ & $<.001^{b}$ & .101 & .234 \\
\hline
\end{tabular}

Note:-WB indicates whole brain; LN, lesion number.

${ }^{\text {a }}$ All data are reported as $P$ values corrected by the Benjamini-Hochberg procedure. Associations between MRI measures were tested by using mixed-model analysis. ${ }^{\mathrm{b}} \mathrm{P}<.05$. 

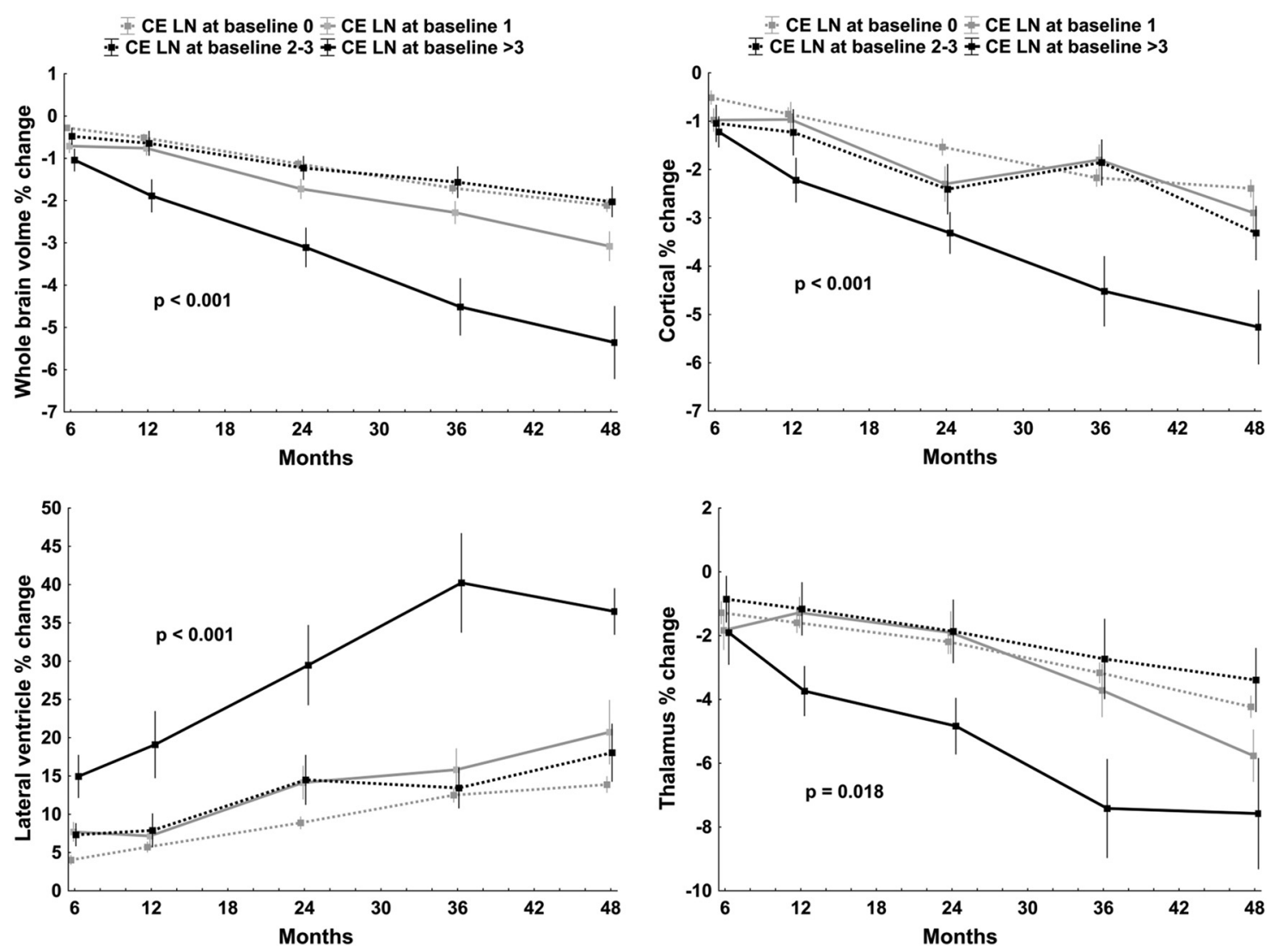

FIG 1. Mixed-effect model analysis of global and tissue-specific brain-volume percent changes over 48 months (dependent variables) and number of CE lesions at baseline (independent variable). For better visualization, the CE lesions were categorized into different baseline number groups, including $0,1,2-3$, and $>3$ lesions. The Benjamini-Hochberg method was used to minimize the false-discovery rate, and $P$ values of $<.05$ were considered significant. ${ }^{23}$

change in SDGM volume was associated with greater baseline T2 $\mathrm{LV}(P=.01)$ and baseline CE lesion number $(P=.013)$.

\section{Mixed-Effect Model Analysis Using Baseline Brain Volumetric Measures as Independent Outcome Variables and Brain-Volume Evolution Measures as Dependent Outcome Variables}

Table 3 shows the relationship between normalized baseline brain-volume measures and associations with their percent changes over 48 months. There were significant associations between decreased individual brain-volume measures at baseline and their greater percent changes over the follow-up period $(P<$ $.05)$. Of all the explored brain-volume measures, only greater enlargement of lateral ventricles showed a consistent association with a decrease in most of the brain-volume measures at baseline $(P<.013)$.

\section{Mixed-Effect Model Analysis Using Evolution of Lesion Measures as Independent Outcome Variables and Brain- Volume Measures as Dependent Outcome Variables}

We performed a mixed-effect model analysis to evaluate the associations between longitudinal changes of lesion variables with respect to brain-volume percent changes over 48 months. The results revealed a significant association between the total cumulative number of new/enlarging T2 lesions and the evolution of whole-brain $(P<.001)$, lateral ventricle $(P=.007)$, GM and thalamic $(P=.013), \operatorname{SDGM}(P=.015)$, and cortical $(P=$
.036) volumes (Table 4 and On-line Fig 1). No significant differences were found between absolute changes in T2 LV (On-line Fig 2), cumulative CE lesion number, or absolute CE LV changes and the evolution of global and tissue-specific brain-volume measures over the 48 months.

The relationship between total cumulative number of new/ enlarging T2 lesions and whole-brain, GM, cortical, lateral ventricle, subcortical deep GM, and thalamic volume changes persisted (all $P<.016$ ), even when adjusting analyses for the baseline brain volume and LV. In this confirmatory analysis, no significant differences were found between absolute changes in T2 LV and the evolution of global and tissue-specific brain-volume measures over the 48 months.

\section{DISCUSSION}

In this prospective, longitudinal, observational study of highrisk patients with CIS for development of clinically definite MS treated with interferon $\beta-1 \mathrm{a},{ }^{5,14-17}$ we investigated the association of brain-volume changes in relation to the formation of new T2 and CE lesions and their volumes over the 48-month study period.

Using mixed-effect model analysis in which global and tissue-specific volumes changes were used as dependent outcome variables and MR imaging lesions at baseline as independent predictors, the progression of brain-volume measures was significantly associated with higher CE and T2 lesion numbers, 

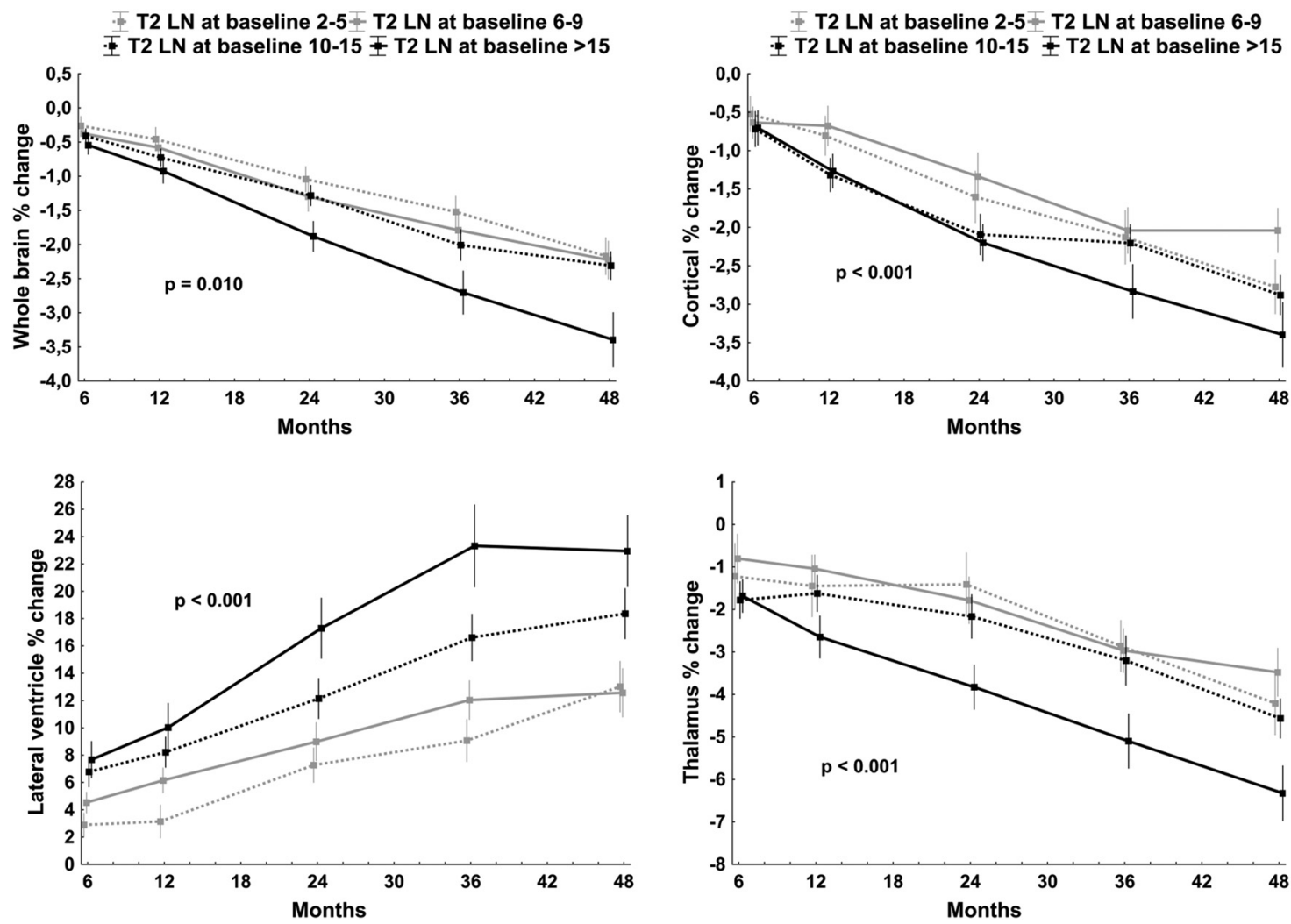

FIG 2. Mixed-effect model analysis of global and tissue-specific brain-volume percent changes over 48 months (dependent variables) and number of $\mathrm{T} 2$ lesions at baseline. For better visualization, the T2 lesions were categorized into different baseline number groups, including 1-5, $6-9,10-15$, and $>15$ lesions. The Benjamini-Hochberg method was used to minimize the false-discovery rate, and $P$ values of $<.05$ are considered significant. ${ }^{23}$

Table 3: Relationship between MRI brain volumetric measures at baseline and evolution of changes over a 48-month period in patients with CIS ${ }^{\mathrm{a}}$

\begin{tabular}{|c|c|c|c|c|c|c|c|}
\hline \multirow{2}{*}{$\begin{array}{l}\text { MRI Brain Volumetric } \\
\text { Measures at Baseline }\end{array}$} & \multicolumn{7}{|c|}{ MRI Brain Volume \% Changes Over 48 mo } \\
\hline & WB & WM & GM & Cortical & Lateral Ventricle & SDGM & Thalamus \\
\hline Normalized WB volume & .712 & $.004^{\mathrm{b}}$ & .426 & .299 & $<.001^{\mathrm{b}}$ & .182 & .174 \\
\hline Normalized WM volume & .591 & $<.001^{\mathrm{b}}$ & .711 & .904 & $.001^{\mathrm{b}}$ & .588 & .254 \\
\hline Normalized GM volume & .881 & .794 & $.049^{\mathrm{b}}$ & .075 & $.004^{\mathrm{b}}$ & .069 & .248 \\
\hline Normalized cortical volume & .847 & .774 & .075 & $.040^{\mathrm{b}}$ & $.007^{\mathrm{b}}$ & .192 & .343 \\
\hline Normalized lateral ventricle volume & .066 & .156 & .935 & .587 & $.013^{\mathrm{b}}$ & .342 & .769 \\
\hline Total normalized SDGM volume & $.032^{\mathrm{b}}$ & .710 & .177 & .291 & .394 & $.042^{b}$ & .457 \\
\hline Normalized thalamic volume & .115 & .578 & .243 & .291 & .343 & $.038^{\mathrm{b}}$ & $<.001^{\mathrm{b}}$ \\
\hline
\end{tabular}

Note:-WB indicates whole brain.

${ }^{\text {a }}$ All data are reported as $P$ values corrected by the Benjamini-Hochberg procedure. Associations between MRI measures were tested by using mixed-model analysis. ${ }^{\mathrm{b}} \mathrm{P}<.05$.

Table 4: Relationship between evolution of MRI lesion and brain volumetric measures over a 48-month period ${ }^{\mathrm{a}}$

\begin{tabular}{|c|c|c|c|c|c|c|c|}
\hline \multirow{2}{*}{$\begin{array}{l}\text { Longitudinal MRI } \\
\text { Lesion Measures }\end{array}$} & \multicolumn{7}{|c|}{ MRI Brain Volume \% Changes Over 48 mo } \\
\hline & WB & WM & GM & Cortical & Lateral Ventricle & SDGM & Thalamus \\
\hline Total cumulative T2 LN & $<.001^{\mathrm{b}}$ & .517 & $.013^{\mathrm{b}}$ & $.036^{\mathrm{b}}$ & $.007^{\mathrm{b}}$ & $.015^{\mathrm{b}}$ & $.013^{b}$ \\
\hline T2 LV absolute change & .115 & .444 & .234 & .456 & .861 & .717 & .659 \\
\hline Total cumulative CE LN & .394 & .182 & .489 & .795 & .341 & .193 & .489 \\
\hline CE LV absolute change & .360 & .936 & .399 & .802 & .160 & .769 & .843 \\
\hline
\end{tabular}

Note:-WB indicates whole brain; LN, lesion number.

${ }^{a}$ All data are reported as $P$ values corrected by the Benjamini-Hochberg procedure. Associations between MRI measures were tested by using mixed-model analysis with interaction with time.

${ }^{\mathrm{b}} \mathrm{P}<.05$.

CE and T2 LVs, and CE positivity. The impact of baseline lesion number and LV on global and tissue-specific brain-volume changes persisted, even when adjusting the analyses for baseline brain volumes. These findings suggest that more se- vere lesion outcomes at baseline are associated with greater development of brain atrophy over the follow-up period in patients with CIS. A possible explanation for these findings is that diffuse inflammation in the WM and GM is responsible 
for accelerated tissue loss that occurs from the earliest disease stages. $^{9,11,13,24}$

Exploring the relationship between accumulation of new/enlarging CE and T2 lesions and global and tissue-specific volumetric changes over 48 months, we found a significant association between the total cumulative number of new/enlarging T2 lesions and the evolution of whole-brain, lateral ventricle, GM, thalamic, SDGM, and cortical volumes. The relationship between lesion accumulation and brain-volume changes over 48 months persisted, even when adjusting the analyses for baseline brain volume and LV. These results suggest that lesion accumulation and brainvolume changes occur simultaneously in the early phase of the disease. Other studies showed an additional correlation between T2 LV and brain-volume changes during early treatment phases. ${ }^{25}$ In our study, however, absolute change of T2 LV was not associated with the progression of brain atrophy measures over the follow-up period, but this finding has to be interpreted with caution, given that over the 48 months of our study, the T2 LV remained stable. Overall, these results suggest that measurement of cumulative accumulation of new/enlarging T2 lesions may be a more sensitive marker of disease activity in patients with CIS than the accumulation of T2 LV.

While the CE lesion number and LV at baseline showed significant associations with the evolution of brain-volume changes over the 48 months, no significant associations between cumulative CE lesions or CE LV absolute changes and the evolution of brain-volume measures over the follow-up period were found in this study. The impact of acute inflammation on the evolution of brain-volume changes remains unclear at this time. ${ }^{26-30}$ The transient nature of CE lesions can be better captured by using frequent serial scanning, which may not be feasible in long-term studies. ${ }^{26,27}$ The findings from this study support the notion that brain atrophy and active inflammation may occur simultaneously, and that monitoring the accumulation of new/enlarging T2 lesions can better reflect this relationship than monitoring CE lesions.

Patients with CIS who presented with lower individual brainvolume measures at baseline showed greater percent changes over the follow-up period. This is an interesting result, because it suggests that, along with the predictive value of baseline lesion burden, the baseline brain volume is also a reliable predictor of future brainvolume changes and therefore of disease progression. ${ }^{31}$ Lower baseline brain volumes found in this study can reflect more aggressive disease ongoing even before the first clinical manifestation. ${ }^{32}$

The results from this study indicate that the association between accumulation of new/enlarging T2 lesions and development of whole-brain atrophy and enlargement of lateral ventricles was stronger than the association with the GM volume measures. The close association between lateral ventricular enlargement and accumulation of T2 lesions in patients with CIS was previously reported. ${ }^{33}$ Other mechanisms at work, including an effect of altered CSF flow pulsatility, may be considered when interpreting these findings. ${ }^{34}$

There is evidence that approximately $40 \%$ of patients with CIS have cortical lesions according to histopathologic examination. ${ }^{35}$ They occur early in patients with CIS and increase in number and size over time. ${ }^{36}$ These lesions are characterized pathologically by demyelination and microglial activation. ${ }^{37,38}$ Therefore, compared with WM lesions, inflammation is not as prominent and breakdown of the blood-brain barrier occurs less frequently with GM lesions. ${ }^{38}$ Assessment of cortical atrophy was proposed as an indirect marker of cortical pathologic assessment over time. ${ }^{4}$ The use of advanced MR imaging sequences, such as double inversion recovery, ${ }^{39}$ can enhance the detection of the number and volume of cortical lesions. We did not apply this sequence; therefore, a somewhat weaker association between accumulation of T2 lesions and the evolution of cortical volume changes in the current study should be interpreted in light of the technical limitations of the MR imaging study protocol.

A strength of this study is the use of a mixed-effect model, which provides the ability to assess the interaction of 2 variables at multiple time points while including covariates. However, there are also several limitations to the present study. It is well known that high-dose intravenous corticosteroid, ${ }^{40}$ interferon $\beta,{ }^{41}$ or natalizumab ${ }^{42}$ treatment leads to a temporary reduction in brain volume, mainly from WM volume loss, ${ }^{42}$ a phenomenon described as pseudoatrophy. ${ }^{43}$ Because all the patients in this study were treated with interferon $\beta$-1a, the rate of brain-volume changes could have been influenced by the anti-inflammatory effect of the drug over the first 6 months of the study period. However, it is less likely that these medications would have impacted significantly on brain-volume changes over the 48 months in the present study. In addition, MR imaging scans were performed at least 30 days after high-dose intravenous corticosteroid administration.

Even though this was a longitudinal serial MR imaging study, it is difficult to establish causality between lesion formation and atrophy progression without using more sophisticated microstructural imaging methods or histopathology; therefore, only inferences about an association are presented. Another approach for determining causes and effects would be to investigate pathologic processes in specific regions, and experimental animal model studies may be more suited for investigation of this relationship. More frequent MR imaging could also provide greater ability to investigate the relationship between lesion accumulation and brain atrophy progression at the earliest clinical stages of MS.

\section{CONCLUSIONS}

We have demonstrated the utility of longitudinal mixed-effect model analysis approaches in the study of patients with CIS, and we have shown that lesion accumulation and brain-volume changes occur simultaneously in the early phase of the disease. More severe LV and lower brain volume at baseline are associated with greater development of brain atrophy over the follow-up period in patients with CIS.

Disclosures: Mihael Varosanec-RELATED: Grant: The SET study was supported by Czech Ministries of Education and Health (NT13237-4/2012, PRVOUK-P26/LF1/4, RVO-VFN64165/2012) and Biogen Idec.* Thomas Uher-UNRELATED: Grants/ Grants Pending: Biogen Idec*; Payment for Lectures (including service on speakers bureaus): Biogen Idec; Travel/Accommodations/Meeting Expenses Unrelated to Activities Listed: Biogen Idec,* Genzyme*; Other: Received financial support for conference travel and honoraria from Biogen Idec. Dana Horakova-RELATED: Grant: The SET study was supported by Czech Ministries of Education and Health (NT13237-4/2012, PRVOUK-P26/LF1/4, RVO-VFN64165/2012) and Biogen Idec*; UNRELATED: Received compensation for travel, speaker honoraria, and consultant fees from Biogen Idec, Novartis, Merck Serono, Bayer Schering, and Teva and support for research activities from Biogen Idec. Michaela Tyblova—RELATED: Grant: The SET study was supported by Czech Ministries of Education and Health (NT132374/2012, PRVOUK-P26/LF1/4, RVO-VFN64165/2012) and Biogen Idec*; UNRELATED: received compensation for travel and honoraria from Biogen Idec, Sanofi Aventis, 
Teva, Novartis, and Merck Serono. Zdenek Seidl-RELATED: Grant: Biogen Idec. Manuela Vaneckova—RELATED: Grant: RVO-VFN64165/2012*; Fees for Participation in Review Activities such as Data Monitoring Boards, Statistical Analysis, Endpoint Committees, and the Like: Biogen Idec; Consultancy: Biogen Idec, Novartis, Merck Serono, and Teva; Grants/Grants Pending: RVO-VFN64165/2012*; Payment for Development of Educational Presentations: Biogen Idec, Novartis, Merck Serono, and Teva; Other: Received compensation for speaker honoraria and consultant fees from Biogen Idec, Novartis, Merck Serono, and Teva and support for research activities from Biogen Idec. Jan Krasensky-RELATED: Support for Travel to Meetings for the Study or Other Purposes: Biogen Idec; Other: Received financial support for research activities from Biogen Idec. Michael Dwyer-UNRELATED: Consultancy: Claret Medical, EMD Serono, Comments: Study consulting by Claret Medical, and served on scientific advisory board for EMD Serono; Grants/Grants Pending: Novartis International, ${ }^{\star}$ Comments: Grant support for new software development. Eva Havrdova—RELATED: Grant: Biogen Idec*; UNRELATED: Consultancy: Biogen Idec, Genzyme, Novartis, Roche, Teva, Merck, and Receptos; Payment for Lectures (including service on speakers bureaus): Biogen Idec, Genzyme, Novartis, Teva, and Merck; Other: Received speaker honoraria and consultant fees from Biogen Idec, Merck Serono, Novartis, Genzyme, and Teva and support for research activities from Biogen Idec and Merck Serono. Robert Zivadinov—RELATED: Grant: Biogen Idec*; UNRELATED: Consultancy: Novartis, Claret Medical, Genzyme, and Teva; Grants/ Grants Pending: Novartis, ${ }^{*}$ Claret Medical, ${ }^{\star}$ Genzyme, ${ }^{\star}$ and Teva*; Payment for Development of Educational Presentations: Genzyme; Other: Received financial support for research activities from Teva Pharmaceuticals, Biogen Idec, Claret Medical, Genzyme, Novartis, and Greatbatch and received personal compensation from Biogen Idec, Novartis, Genzyme, Claret Medical, and EMD Serono for speaking and consultant services. *Money paid to the institution.

\section{REFERENCES}

1. Confavreux C, Vukusic S. Natural history of multiple sclerosis: a unifying concept. Brain 2006;129:606-16

2. Zivadinov R, Bakshi R. Role of MRI in multiple sclerosis II: brain and spinal cord atrophy. Front Biosci 2004;9:647-64

3. Miller DH, Barkhof F, Frank JA, et al. Measurement of atrophy in multiple sclerosis: pathological basis, methodological aspects and clinical relevance. Brain 2002;125:1676-95

4. Geurts JJ, Stys PK, Minagar A, et al. Gray matter pathology in (chronic) MS: modern views on an early observation. J Neurol Sci 2009;282:12-20

5. Zivadinov R, Havrdová E, Bergsland $\mathrm{N}$, et al. Thalamic atrophy is associated with development of clinically definite multiple sclerosis. Radiology 2013;268:831-41

6. Calabrese M, Rinaldi F, Mattisi I, et al. The predictive value of gray matter atrophy in clinically isolated syndromes. Neurology 2011;77:257-63

7. Dalton CM, Chard DT, Davies GR, et al. Early development of multiple sclerosis is associated with progressive grey matter atrophy in patients presenting with clinically isolated syndromes. Brain 2004;127:1101-07

8. Raz E, Cercignani M, Sbardella E, et al. Gray- and white-matter changes 1 year after first clinical episode of multiple sclerosis: MR imaging. Radiology 2010;257:448-54

9. Fisniku LK, Chard DT, Jackson JS, et al. Gray matter atrophy is related to long-term disability in multiple sclerosis. Ann Neurol 2008;64:247-54

10. Zivadinov R, Bergsland N, Dolezal O, et al. Evolution of cortical and thalamus atrophy and disability progression in early relapsing-remitting $\mathrm{MS}$ during 5 years. AJNR Am J Neuroradiol 2013;34:1931-39

11. Fisher E, Lee JC, Nakamura K, et al. Gray matter atrophy in multiple sclerosis: a longitudinal study. Ann Neurol 2008;64:255-65

12. Cappellani R, Bergsland N, Weinstock-Guttman B, et al. Subcortical deep gray matter pathology in patients with multiple sclerosis is associated with white matter lesion burden and atrophy but not with cortical atrophy: a diffusion tensor MRI study. AJNR Am J Neuroradiol 2014;35:912-19

13. Roosendaal SD, Bendfeldt K, Vrenken $H$, et al. Grey matter volume in a large cohort of MS patients: relation to MRI parameters and disability. Mult Scler 2011;17:1098-106

14. Kalincik T, Vaneckova M, Tyblova M, et al. Volumetric MRI markers and predictors of disease activity in early multiple sclerosis: a longitudinal cohort study. PLoS ONE 2012;7:e50101

15. Uher T, Benedict RH, Horakova D, et al. Relationship between gray matter volume and cognitive learning in CIS patients on diseasemodifying treatment. J Neurol Sci 2014;347:229-34

16. Uher T, Blahova-Dusankova J, Horakova D, et al. Longitudinal MRI and neuropsychological assessment of patients with clinically isolated syndrome. J Neurol 2014;261:1735-44

17. Uher T, Horakova D, Bergsland N, et al. MRI correlates of disability progression in patients with CIS over 48 months. Neuroimage Clin 2014;6:312-19

18. Zivadinov R, Heininen-Brown M, Schirda CV, et al. Abnormal subcortical deep-gray matter susceptibility-weighted imaging filtered phase measurements in patients with multiple sclerosis: a case-control study. Neuroimage 2012;59:331-39

19. Smith SM, Zhang Y, Jenkinson M, et al. Accurate, robust, and automated longitudinal and cross-sectional brain change analysis. $\mathrm{Neu}$ roimage 2002;17:479-89

20. Patenaude B, Smith SM, Kennedy DN, et al. A Bayesian model of shape and appearance for subcortical brain segmentation. Neuroimage 2011;56:907-22

21. Smith SM, De Stefano N, Jenkinson M, et al. Normalized accurate measurement of longitudinal brain change. J Comput Assist Tomogr 2001;25:466-75

22. Dwyer MG, Bergsland N, Zivadinov R. Improved longitudinal gray and white matter atrophy assessment via application of a 4-dimensional hidden Markov random field model. Neuroimage 2014;90:207-17

23. Benjamini Y, Drai D, Elmer G, et al. Controlling the false discovery rate in behavior genetics research. Behav Brain Res 2001;125:279-84

24. Calabrese M, Atzori M, Bernardi V, et al. Cortical atrophy is relevant in multiple sclerosis at clinical onset. J Neurol 2007;254:1212-20

25. Comi G, Martinelli V, Rodegher M, et al. Effects of early treatment with glatiramer acetate in patients with clinically isolated syndrome. Mult Scler 2013;19:1074-83

26. Zivadinov R, Zorzon M. Is gadolinium enhancement predictive of the development of brain atrophy in multiple sclerosis? A review of the literature. J Neuroimaging 2002;12:302-09

27. Zivadinov R, Bagnato F, Nasuelli D, et al. Short-term brain atrophy changes in relapsing-remitting multiple sclerosis. J Neurol Sci 2004;223:185-93

28. Rashid W, Davies GR, Chard DT, et al. Relationship of triple dose contrast enhanced lesions with clinical measures and brain atrophy in early relapsing-remitting multiple sclerosis: a two-year longitudinal study. Mult Scler 2007;13:178-85

29. Inglese M, Mancardi GL, Pagani E, et al. Brain tissue loss occurs after suppression of enhancement in patients with multiple sclerosis treated with autologous haematopoietic stem cell transplantation. J Neurol Neurosurg Psychiatry 2004;75:643-44

30. Paolillo A, Piattella MC, Pantano P, et al. The relationship between inflammation and atrophy in clinically isolated syndromes suggestive of multiple sclerosis: a monthly MRI study after triple-dose gadolinium-DTPA. J Neurol 2004;251:432-39

31. De Stefano N, Giorgio A, Battaglini M, et al. Assessing brain atrophy rates in a large population of untreated multiple sclerosis subtypes. Neurology 2010;74:1868-76

32. Rojas JI, Patrucco L, Míguez J, et al. Brain atrophy in radiologically isolated syndromes. J Neuroimaging 2015;25:68-71

33. Dalton CM, Brex PA, Jenkins R, et al. Progressive ventricular enlargement in patients with clinically isolated syndromes is associated with the early development of multiple sclerosis. J Neurol Neurosurg Psychiatry 2002;73:141-47

34. Magnano C, Schirda C, Weinstock-Guttman B, et al. Cine cerebrospinal fluid imaging in multiple sclerosis. J Magn Reson Imaging 2012;36:825-34

35. Lucchinetti CF, Popescu BF, Bunyan RF, et al. Inflammatory cortical demyelination in early multiple sclerosis. N Engl J Med 2011;365:2188-97 
36. Calabrese M, Rocca MA, Atzori M, et al. Cortical lesions in primary progressive multiple sclerosis: a 2-year longitudinal MR study. Neurology 2009;72:1330-36

37. Bø L, Vedeler CA, Nyland $\mathrm{H}$, et al. Intracortical multiple sclerosis lesions are not associated with increased lymphocyte infiltration. Mult Scler 2003;9:323-31

38. van Horssen J, Brink BP, de Vries HE, et al. The blood-brain barrier in cortical multiple sclerosis lesions. J Neuropathol Exp Neurol 2007;66:321-28

39. Geurts JJ, Pouwels PJ, Uitdehaag BM, et al. Intracortical lesions in multiple sclerosis: improved detection with 3D double inversionrecovery MR imaging. Radiology 2005;236:254-60
40. Fox RJ, Fisher E, Tkach J, et al. Brain atrophy and magnetization transfer ratio following methylprednisolone in multiple sclerosis: short-term changes and long-term implications. Mult Scler 2005;11:140-45

41. Hardmeier M, Wagenpfeil S, Freitag P, et al. Rate of brain atrophy in relapsing MS decreases during treatment with IFNbeta-1a. Neurology 2005; $64: 236-40$

42. Vidal-Jordana A, Sastre-Garriga J, Pérez-Miralles F, et al. Early brain pseudoatrophy while on natalizumab therapy is due to white matter volume changes. Mult Scler 2013;19:1175-81

43. Zivadinov R, Reder AT, Filippi M, et al. Mechanisms of action of disease-modifying agents and brain volume changes in multiple sclerosis. Neurology 2008;71:136-44 